\title{
Delay Sensitivity Based Assignment of OVSF Codes for the Downlink DCH in WCDMA
}

\author{
Dimitrios N. Skoutas and Angelos N. Rouskas \\ Department of Information and Communication Systems Engineering, \\ University of the Aegean, Samos 83200, Greece \\ \{d.skoutas, arouskas\}@aegean.gr
}

\begin{abstract}
A significant problem that deteriorates the performance of 3G W-CDMA systems is code blocking. The complete elimination of this phenomenon is accomplished only if a code reassignment procedure is employed. However, the relatively high total signaling delay for a single OVSF code reassignment combined with the strict delay requirements of some services makes the reassignment of some OVSF codes impractical. In other words, that a reassignment procedure cannot always be performed and therefore code blocking cannot be eliminated. In this paper, we introduce a new code selection scheme, named Delay Sensitivity Based Assignment (DSBA), which takes into account the different delay requirements of the incoming calls. The performance of the proposed scheme is evaluated through event driven simulation and the results show a significant decrease in code blocking probability, especially for high rate calls, compared to other previously proposed schemes.
\end{abstract}

\section{Introduction}

In third generation mobile communication systems, WCDMA has been selected as the most promising technology to support high data rate and variable data rate services with different QoS requirements. Multiple rate transmission is supported by using Orthogonal Variable Spreading Factor (OVSF) codes as channelisation codes [1],[2].

At the downlink of (UTRA FDD) WCDMA systems, a constant bit rate service is transmitted through a Dedicated Channel $(\mathrm{DCH})$ while a variable bit rate service is transmitted through a combination of a $\mathrm{DCH}$ and a Downlink Shared Channel (DSCH) or a DCH and a High Speed-DSCH channel. Each channel is assigned an OVSF code, which in the case of the downlink DCH channel is normally fixed for the duration of the connection [4].

On the other hand, due to code blocking, it is often necessary to reallocate a downlink DCH from one OVSF code to another in order to accommodate a new call. However, if the corresponding connection has very strict delay requirements the downlink DCH may be "unmovable" and cannot be reallocated to another OVSF code. Therefore, a reassignment procedure may not be successful due to the unmovable DCH channels. Thus, code blocking phenomenon cannot always be eliminated, even if a code reassignment procedure is employed along with an OVSF code assignment scheme. 
Previous works on the reduction of code blocking are attempting to keep the code tree less fragmented, but they do not differentiate between movable and unmovable DCH channels [3], [9], [10]. Consequently, they allow the spreading of the unmovable DCH channels across the OVSF code tree. That decreases the efficiency of the code reassignment procedure, which in turn increases the code blocking probability especially for high rate calls.

In this paper, we propose a new code selection scheme, named Delay Sensitivity Based Assignment (DSBA). DSBA tries to keep the allocation of the unmovable DCH channels as compact as possible at the OVSF code tree. Thus, a code reassignment procedure is more efficient and the code-blocking phenomenon is reduced compared to previously proposed schemes.

The rest of the paper is organized as follows. The system model is presented in Section 2. In Section 3, the problem statement is outlined. Section 4 presents an overview of previously proposed schemes. The DSBA scheme is described in Section 5. Section 6 presents the numerical results and Section 7 concludes our study.

\section{System Description}

A detailed description on OVSF code generation can be found in [1], [2]. The OVSF code tree, shown in Figure 1, is a binary tree where each node represents a channelisation code. Each code can be denoted as $C_{S F, k}$, where $S F$ is the spreading factor of the code and $k$ is the code number, $1 \leq k \leq S F$. The higher the spreading factor the lower the transmission rate supported by a code. Leaf codes have the maximum spreading factor $\left(S F_{\max }\right)$ and therefore the minimum data rate, which is denoted by $R$. The transmission rate $R_{S}$ supported by an OVSF code with spreading factor $S F$ is always a multiple of a power of two of the lowest available rate:

$$
R_{S}=K \times R, K=\frac{S F_{\max }}{S F}=1,2,4,8, \ldots
$$

Two OVSF codes are orthogonal, if and only if, none of them is an ancestor of the other. Therefore, once a code is assigned, all of its ancestors, as well as all of its descendants are blocked and cannot be used until the code is released.

\subsection{Code Blocking Condition}

A difficulty in the assignment of OVSF codes is a condition called code blocking [3]. An example is shown in Figure 1. Suppose that codes $C_{41}$ and $C_{44}$ are already assigned. Due to the orthogonality constraint, all the descendant and ancestor codes of $C_{41}$ and $C_{44}$ respectively cannot be used. Although the system has adequate capacity to support an additional connection at rate $8 \mathrm{R}$ the only codes that can support rate $8 \mathrm{R}\left(C_{21}\right.$ and $\left.C_{22}\right)$ are already blocked.

Code blocking reduces spectral efficiency and increases call-blocking probability especially for higher data rate users. A first possible countermeasure that reduces this phenomenon is the clever selection among possible candidate codes 


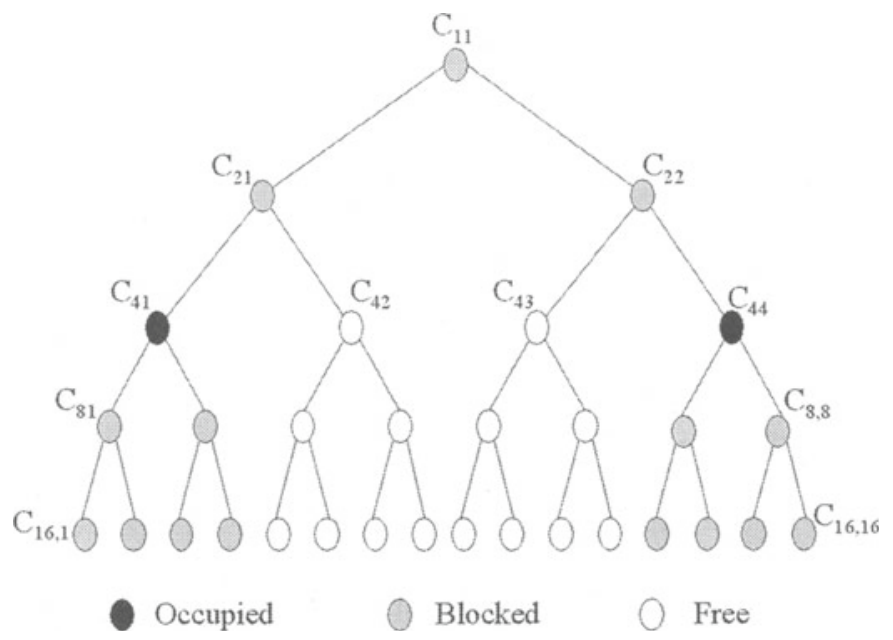

Fig. 1. Code blocking: although the total remaining capacity is $8 \mathrm{R}$, a connection of rate $8 \mathrm{R}$ will be blocked

during the assignment process. However, because of the statistical nature of the departure process, the complete elimination of code blocking is accomplished only if a code reassignment procedure can be employed. A reassignment procedure reallocates ongoing calls to other codes so that a new call can always be supported if the system has enough free capacity to support the requested rate.

\subsection{Transport Channels}

In (UTRA FDD) WCDMA [4], there are three types of downlink transport channels:

1. Common channels are suited for the transmission of small data amounts such as signaling data or small IP packets. Therefore, common channels are mainly used during connection set up process.

2. Shared channels allow a single OVSF channelization code to be shared among several users. They are made for the transmission of medium or large data amounts. There are two types of downlink shared channels:

a) The Downlink Shared Channel (DSCH) and

b) The High Speed Downlink Shared Channel (HS-DSCH).

DSCH and HS-DSCH may change transmission rate every $10 \mathrm{~ms}$ or $2 \mathrm{~ms}$ respectively. Both DSCH and HS-DSCH channels are associated with a DCH.

3. Dedicated channels are reserved for a single user only and support transmission rates from a few kbps up to $2 \mathrm{Mbps}$. Each downlink DCH channel is assigned an OVSF code, which is normally fixed for the duration of the connection. Downlink DCH is used in the following cases [4], [5]:

a) For conversational Real Time (RT) services that have fairly constant bit rate but high delay sensitivity.

b) In association with a DSCH Channel.

c) In association with a HS-DSCH Channel. 


\section{Problem Statement}

Code blocking can be easily solved in the case of DSCH or HS-DSCH channels since for these channels a reassignment procedure can always be performed every $10 \mathrm{~ms}$ or $2 \mathrm{~ms}$ respectively. This is not the case for the downlink DCH channels: The total signaling delay for a single OVSF code reassignment, performed with an RRC layer procedure called Physical Channel Reconfiguration, is evaluated at $220 \mathrm{~ms}$ [6]. Such a delay can not be tolerated by Real Time (RT) conversational services which in most cases have very strict delay limits (around 250ms) [7], [8] but can be tolerated by an interactive service such as Web- browsing.

We will refer to a DCH channel as "unmovable" if it is used by an RT conversational service, or if it is associated with a HS-DSCH, which is used by some service with very strict delay limit. Then in either of these cases, DCH cannot be reassigned. That is because such a reassignment would significantly increase the total delay, resulting in unacceptable quality of service.

On the other hand, we will refer to a DCH channel as "movable" if it is related with a delay insensitive service. For example, there is no restriction when reassigning a downlink DCH associated with a DSCH, as the applications served by the latter usually have very low delay sensitivity.

Concluding, the problem at hand is that the reassignment procedure is impossible when some of the involved OVSF codes cannot be reassigned, and hence code blocking cannot be eliminated. In the rest of the paper, we will refer to these codes, related to an unmovable DCH, as the "unmovable" codes. On the other hand, we will refer to the codes related to some service with low delay sensitivity, as the "movable" codes since these codes can be reassigned (e.g. codes assigned to a $\mathrm{DCH}$ associated with a DSCH).

\section{Overview of Other Proposed Schemes}

\subsection{Dynamic Code Assignment (DCA)}

A dynamic code assignment (DCA) scheme is proposed in [3]. DCA aims at minimizing the number of OVSF codes that must be reassigned in order to support a new call. The algorithm is based on the concept of assigning a cost function to each candidate branch and identifying a branch with a minimal cost. The cost of a branch is defined as the minimum number of code reassignments, which are necessary in order to empty the branch. This scheme does not specify a code allocation strategy when a new call arrives or when an ongoing call is reallocated. If there are more than one candidate codes for an incoming call then any of them can be assigned to the new call. The complexity of the DCA scheme depends on the type of the search algorithm used to locate the minimum cost branch.

\subsection{Crowded-First Scheme}

Crowded-first strategy is proposed in [9]. The main idea behind this scheme is to keep the code tree less fragmented by allocating the codes as compact as 
possible. This is achieved by always selecting the code, whose immediate upper layer subbranch will have the least free capacity after the allocation. In the case of ties the search continues one more layer up, until the tie is resolved. Crowdedfirst is very efficient and significantly reduces code-blocking probability provided that all the DCH channels are allocated to movable OVSF codes.

\subsection{Fewer Codes Blocked (FCB) Scheme}

In [10] the Fewer Codes Blocked (FCB) scheme is introduced. In this scheme the measure that differentiates the candidate codes during the selection process is the number of upper layer codes that are not already blocked due to some other existing code allocation and will be blocked if the candidate code is assigned. The code, which has the minimum value according to the FCB criterion, is the one to be selected. Ties are resolved by choosing the first, from the left, code of the OVSF code tree, which supports a rate equal to the requested rate (Leftmost criterion). FCB has the same efficiency as the crowded-first scheme, but it has less computational complexity. That is because FCB does not require a recursive search from the level of the code up to the root level during decision process.

During system operation it is inevitable that the OVSF code tree will become fragmented regardless of the code assignment scheme. Therefore, a code assignment scheme has always to be combined with a reassignment procedure in order to eliminate code blocking. Because FCB and Crowded-first schemes use the same criterion for the allocation of both movable and unmovable OVSF codes, the unmovable codes are scattered across the code tree. Consequently, a reassignment procedure may fail due to code blocking, and unmovable OVSF codes must be allocated as compact as possible at the OVSF code tree.

\section{The Delay Sensitivity Based Assignment Scheme}

Assuming that all the connections are served by DCH channels a code at the OVSF code tree may be a:

1. Free code: The code and all of its ancestors as well as all of its descendant codes are not assigned to a downlink DCH channel. (e.g. $C_{82}$ in Figure 2)

2. Occupied movable code: The code is assigned to a movable downlink DCH channel. (e.g. $C_{86}$ in Figure 2)

3. Occupied unmovable code: The code is assigned to an unmovable downlink DCH channel. (e.g. $C_{81}$ in Figure 2)

4. Blocked movable code: One of its ancestors or one or more of its descendant codes is assigned to a movable downlink DCH channel. (e.g. $C_{43}$ in Figure 2)

5. Blocked unmovable code: One of its ancestors or one or more of its descendant codes is assigned to an unmovable downlink DCH channel. (e.g. $C_{41}$ in Figure 2)

The main idea behind the proposed Delay Sensitivity Based Assignment (DSBA) scheme is to avoid the scattering of the unmovable OVSF codes across 
the code tree. That is accomplished with clever selection among possible candidate codes during the assignment process. In that way, the use of a code reassignment procedure will be successful more frequently and code blocking phenomenon will be reduced. The proposed scheme consists of two sub-schemes namely M-FCB and U-FCB. More specifically, at the arrival of a new call requesting a rate of $k R$, where $k$ is a power of two:

1. IF the system has enough capacity to support the rate requirement of the call then:

a) IF the service has low delay sensitivity, it will be allocated to a movable code and the M-FCB scheme will be used.

b) ELSE the service has high delay sensitivity, it will be allocated to an unmovable code and the U-FCB scheme will used.

2. ELSE the call is blocked

\subsection{The M-FCB Scheme}

Candidate codes to receive the new call are all the free codes that support a rate of $k R$.

1. IF there is one or more candidate codes: The criterion for the selection of a candidate code is the number of upper layer codes that are not already blocked by some other movable codes and will be blocked if the candidate code is assigned. The code that has the minimum value according to this criterion is the one to be selected. Ties are resolved by choosing the first, from the right, candidate code of the OVSF code tree, which supports a rate equal to the requested rate.

2. ELSE the Code Reassignment Scheme is applied. If the reassignment scheme fails due to code blocking, the call is blocked.

\subsection{Code Reassignment Scheme}

Code Reassignment Scheme is a heuristic algorithm, which aims at minimizing the number of OVSF codes that must be reassigned in order to support a new call. Candidate codes to receive the new call are those codes that are restrained from use because some of their descendants are occupied only by movable OVSF codes.

1. IF there is one or more candidate codes:

a) Sort the candidate codes according to the number of their occupied descendant codes and choose the one with the smallest number. Resolve ties by selecting the code whose occupied descendant codes carry the smallest capacity.

b) Once the subbranch to become empty is selected, each of the occupied descendant codes must be transferred to other subbranches. Each reassignment is treated like a new call. However, a reassignment may fail due to code blocking. 


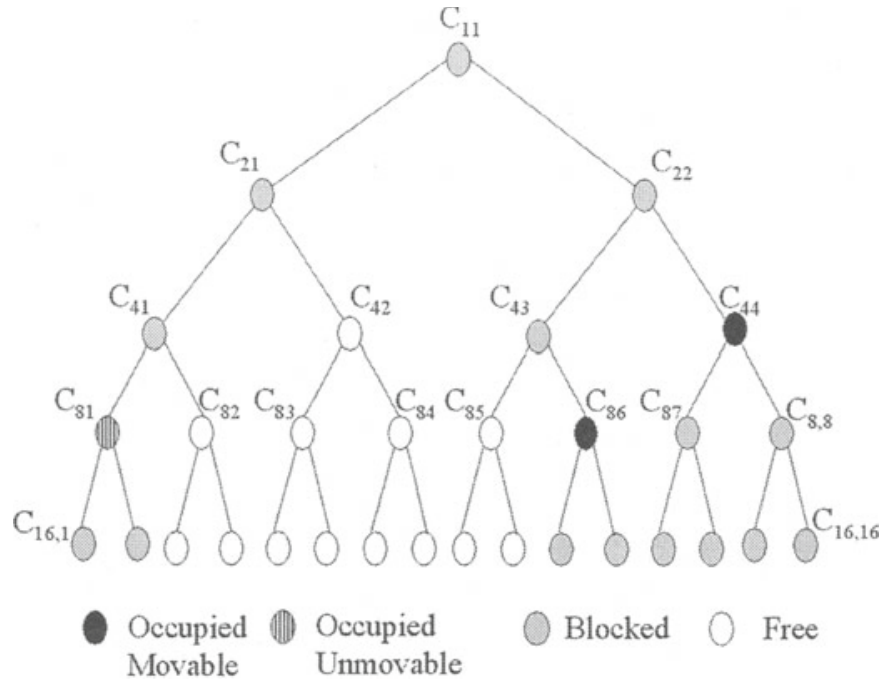

Fig. 2. Code assignment examples

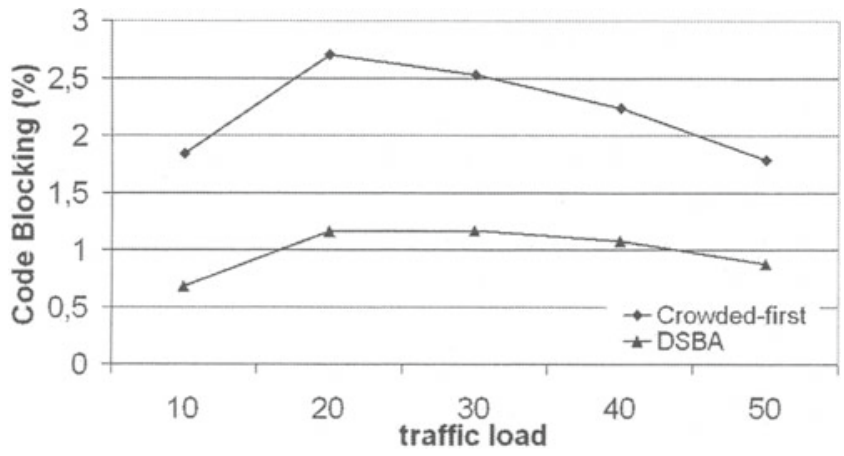

Fig. 3. Code blocking probability when percentage of unmovable codes is low

i. IF all the reassignments are completed successfully the candidate code is assigned to the new call.

ii. ELSE the candidate code is rejected and the procedure continues from step 1 for the rest of the candidate codes.

2. ELSE the call is blocked.

\subsection{The U-FCB Scheme}

From all the OVSF codes of rate equal to the requested rate, the candidates codes to receive the new call are:

- The free OVSF codes.

- The occupied movable OVSF codes. 
- The OVSF codes which are blocked only by some other lower rate movable codes.

1. IF there is one or more candidate codes:

a) The criterion for the selection of a candidate code is the number of its ancestor codes that will be blocked after the code assignment and they are not already blocked by some other unmovable codes. The code that has the minimum value according to this criterion is the one to be selected. Ties are resolved by choosing the first from the left candidate code at the OVSF code tree.

b) If the chosen candidate code is already blocked or occupied by movable codes then the codes that cause the blocking must be transferred to other subbranches. If any of the reassignments fail due to code blocking then the candidate code is rejected and U-FCB criterion is again applied to the rest of the candidate codes. The code assignment process concludes when an appropriate code is found or when all the candidate codes are rejected.

2. ELSE the call is blocked

For example consider the code tree in Figure 2. Suppose a new call requesting a code of rate $2 R$ :

1. If the user's service is not delay sensitive then the OVSF code, which will be allocated to the call, will be a movable code. In this case the M-FCB scheme will be used in order to find the most appropriate OVSF code for the call. According to the M-FCB scheme candidate codes for the new call are $C_{82}, C_{83}, C_{84}$ and $C_{85}$. The value of the M-FCB criterion for the $C_{82}$, $C_{83}, C_{84}$ and $C_{85}$ codes are respectively $2,2,2$ and 0 . Consequently code $C_{85}$, which has the minimum value according to M-FCB criterion is the one to be selected.

2. If the user's service is very delay sensitive then the OVSF code, which will be allocated to the call, will be an unmovable code. In this case the U-FCB criterion will be used in order to find the most appropriate OVSF code for the call. According to the U-FCB criterion candidate codes for the new call are $C_{82}, C_{83}, C_{84}, C_{85}$ and $C_{86}$ and the values of the U-FCB criterion are respectively $0,1,1,2$ and 2 . Consequently code $C_{82}$, which has the minimum value of the U-FCB criterion will be selected.

Concluding we can say that the main idea behind the DSBA scheme is to allocate a movable code closer to other movable codes and an unmovable code closer to other unmovable codes. In that way, the scattering of unmovable codes along the code tree is avoided and therefore code blocking is reduced.

\section{$6 \quad$ Numerical Results}

The performance of the proposed scheme is evaluated through event driven simulation on a 7-layer OVSF code tree. Calls are assumed to arrive according to 


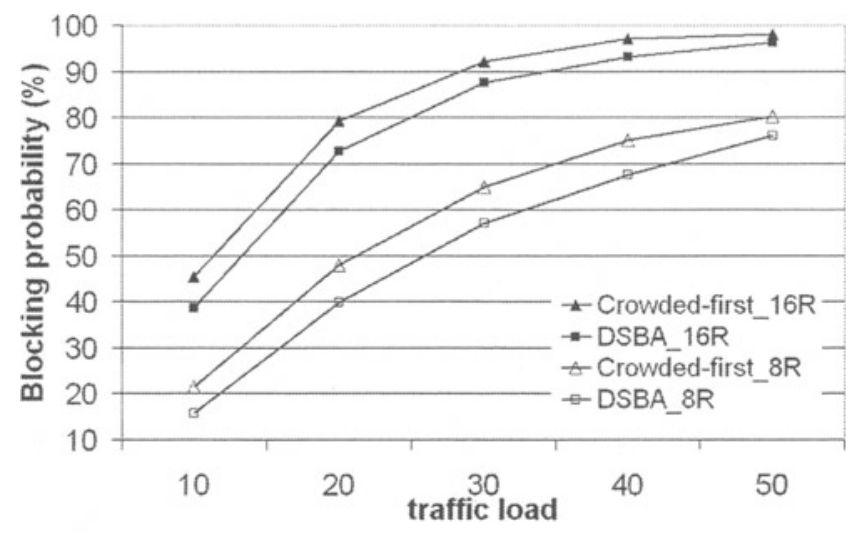

Fig. 4. Blocking probability of high rate calls when percentage of unmovable codes is low

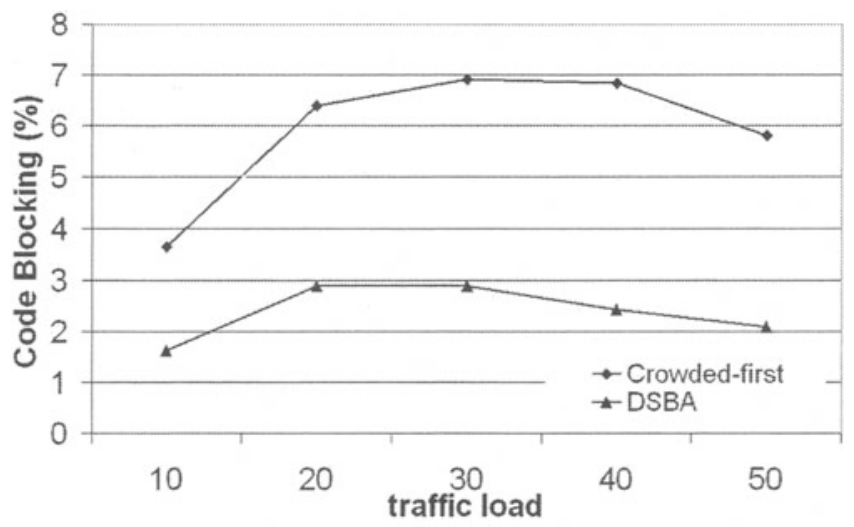

Fig. 5. Code blocking probability when percentage of unmovable codes is high

a Poisson process, while their duration is exponentially distributed with equal mean. We assume that all the connections are served by DCH channels. Each incoming call may request a rate of $R, 2 R, 4 R, 8 R$, or $16 R$ and has a $P_{U}$ probability to be related with a delay sensitive service with a very strict delay limit. In the last case, the incoming call will be allocated to an unmovable OVSF code. To ensure the stability of the results, each simulation run consisted of at least 100000 incoming calls.

The goal of the simulation is to study the code blocking performance of DSBA scheme in two different study scenarios. Crowded-first scheme combined with a Code Reassignment Scheme has also been evaluated for comparative purposes. The uniform rate distribution $(R: 2 R: 4 R: 8 R: 16 R=20: 20: 20: 20: 20)$ is used in both scenarios while the $P_{U}$ probability changes. 


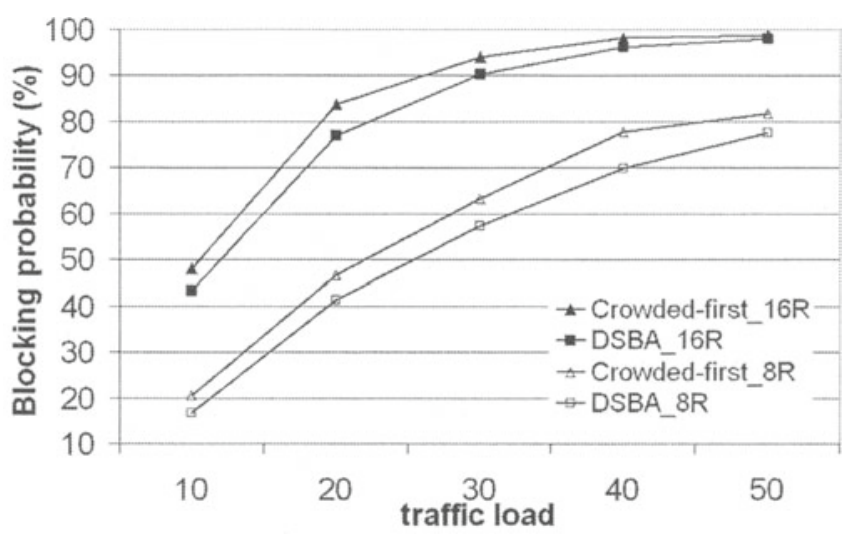

Fig. 6. Blocking probability of high rate calls when percentage of unmovable codes is high

\subsection{Low Percentage of Unmovable Codes}

At the first study scenario $P_{U}=0,4$. The code blocking probability for increasing traffic load is shown in Figure 3. For both schemes as the traffic load increases, the code blocking probability reaches its peak value and then, as the traffic load continues to increase, the code blocking probability decreases. That is because at high traffic loads the calls often cannot be served by the system due to lack of capacity (capacity blocking). Therefore, capacity blocking gradually replaces code blocking which is reduced. When DSBA is used, it can be observed that the code blocking probability is very low and ranges approximately between 0,7 and 1,2. This is not the case for the Crowded-first scheme. The scattering of the unmovable OVSF codes across the code tree leads to higher code blocking probability, which ranges approximately between 1,8 and 2,7. Calls requesting for high rates are the most affected by code blocking. By reducing code blocking, the blocking rate for such calls is reduced and therefore call acceptance becomes more fair. Figure 4 shows the blocking rate of calls requesting for an $8 R$ or $16 R$ rate under the DSBA and Crowded-fist schemes respectively. As we can observe the use of the DSBA scheme results in reduced blocking rate at all traffic loads.

\subsection{High Percentage of Unmovable Codes}

At the second study scenario the percentage of unmovable OVSF codes is increased to $P_{U}=0,6$. Figure 5 shows the code blocking probability for increasing traffic load. As we expected, the increased number of unmovable OVSF codes results in increased code blocking for both schemes. However, the code blocking probability for the DSBA scheme does not exceed 3 per cent while at the same time for the Crowded-first scheme the code blocking probability ranges approximately between 3.8 and 7. Finally, Figure 6 shows the blocking rate for calls requesting for an $8 R$ or $16 R$ rate. As in the first study scenario, DSBA scheme outperforms Crowded-first at all traffic loads. 


\section{Conclusions}

We proposed a dynamic OVSF code allocation scheme namely Delay Sensitivity Based Assignment (DSBA), for the downlink DCH channel of W-CDMA 3G mobile communication systems. DSBA takes into account the different delay requirements of the incoming calls, reduces code blocking and consequently reduces the blocking rate especially for higher rate calls, which are most affected otherwise. Therefore, call acceptance becomes fairer. The simulation results have demonstrated the efficiency of the DSBA scheme compared to some other previously published approach.

\section{References}

1. F. Adashi, M. Sawahashi, and K. Okawa: Tree structured generation of orthogonal spreading codes with different lengths for forward link of DS-CDMA mobile radio. Electronic Letters, vol. 33, pp. 27-28, Jan 1997.

2. 3GPP, Technical Specification 25.213 V5.2.0 (2002-09), Spreading and Modulation (FDD).

3. T. Minn, K.Y. Siu: Dynamic Assignment of Orthogonal Variable Spreading Factor Codes in W- CDMA. IEEE Journal on Selected Areas in Communications, Vol. 18, No. 8, August 2000, pages 1429-1440.

4. Harri Holma and Antti Toskala: WCDMA for UMTS Radio Access For Third Generation Mobile Communications. John Wiley and Sons, 2001.

5. 3GPP, Technical Specification TR 25.848 V4.0.0 (2001-03), Physical layer aspects of UTRA High Speed Downlink Packet Access.

6. Paolo Goria, Claudio Guerrini, Alessandro Vaillant: Signaling delay of code allocation strategies. In Proc. of IST Mobile \& Wireless Telecommunications Summit 2002.

7. 3GPP Technical Specification 22.105 V6.2.0 (2003-2006) Services and service capabilities.

8. 3GPP Technical Report 25.853 V4.0.0 (2001-2003) Delay Budget within the Access Stratum.

9. Y.-C. Tseng, C.-M. Chao, and S.-L. Wu: Code placement and replacement strategies for wideband CDMA OVSF code tree management. in Proc. of IEEE GLOBECOM, vol. 1, pp. 562-566, 2001.

10. A. Rouskas and D. Skoutas: OVSF code assignment and reassignment at the forward link of W- CDMA 3G systems. In Proc. of IEEE PIMRC, vol. 5, pp. 24042408,2002 